\title{
Voxel-based analysis for identification of urethro-vesical subregions predicting urinary toxicity after prostate cancer radiotherapy
}

\begin{abstract}
Eugenia Mylona $(\mathrm{MSc})^{1}{ }^{1}{ }^{*}$, Oscar Acosta $(\mathrm{PhD})^{1}$, Thibaut Lizee $(\mathrm{MD})^{1}$, Caroline Lafond $(\mathrm{PhD})^{1}$, Gilles Crehange $(M D, P h D)^{2}$, Nicolas Magné $(M D, P h D)^{3}$, Sophie Chiavassa $(P h D)^{4}$, Stéphane Supiot $(M D, P h D)^{4}$, Juan David Arango Ospina (PhD) ${ }^{1}$, Borris Campillo-Gimenez $(\mathrm{MD})^{1}$, Joel Castelli (MD, PhD) ${ }^{1}$, Renaud de Crevoisier (MD, PhD) ${ }^{1}$
\end{abstract}

${ }^{1}$ Univ Rennes, CLCC Eugène Marquis, INSERM, LTSI - UMR 1099, F-35000 Rennes, France.

${ }^{2}$ Department of Radiation Oncology, Centre Georges François Leclerc, Dijon, France.

${ }^{3}$ Department of Radiation Oncology, Lucien Neuwirth Cancer Institute, St Priest en Jarez, France.

${ }^{4}$ Department of Radiation Oncology, Institut de Cancérologie de I'Ouest, Saint Herblain, France.

\section{Corresponding author}

Renaud DE CREVOISIER,

Radiation Department, Eugene Marquis' Comprehensive Cancer Center, LTSI, INSERM 1099, Rennes University, Rue de la Bataille Flandres Dunkerque CS 44229, 35042 Rennes Cedex, France

Tel : 0033637395413 ; Fax : 0033299253033

r.de-crevoisier@rennes.unicancer.fr

\section{Contributors}


RdC, EM and OA designed the study. RdC was the principal investigator of the STIC-IGRT trial. SS was the principal investigator of the French cohort belonging to the PROFIT trial. RdC, SS, CL, NM and SC recruited and treated patients. EM, OA, TL, JC and RdC performed the image processing and the statistical analysis. All authors interpreted data, wrote and reviewed the manuscript.

\section{Short running tittle}

Voxel-based analysis predicting urinary toxicity

\section{Declaration of interests}

We declare no competing interests.

\section{Acknowledgements}

This work was supported by the Institut National du Cancer (INCa) for the STIC IGRT-P and PROFIT trials, and received a French government grant (through the CominLabs excellence laboratory and managed by the National Research Agency in the "Investing for the Future" program, under reference ANR-10-LABX07-01). We also thank Marion Renouf for her help to collect the data.

This phrase was blinded in the manuscript and the ansers to the reviewers:

The trials were approved by the French Institutional Review Board and are registered in ClinicalTrials.gov (NCT00433706 for the STIC-IGRT trial, NCT00304759 for the PROFIT trial). 


\section{ABSTRACT}

Background and purpose: To apply a voxel-based analysis to identify urethro-vesical symptomrelated subregions (SRSs) associated with acute and late urinary toxicity in prostate cancer radiotherapy.

Materials and methods: A total of 272 prostate cancer patients treated with IMRT/IGRT were prospectively analyzed. Each patient's CT was spatially normalized to a common coordinate system (CCS) via nonrigid registration. The obtained deformation fields were used to map the dose of each patient to the CCS. A voxel-based statistical analysis was applied to generate 3D dose-volume maps for different urinary symptoms allowing the identification of corresponding SRSs where statistically significant dose differences between patients with/without toxicity. Each SRS was propagated back to each individual's native space and DVHs for the SRSs and the whole bladder were computed. Logistic and Cox regression were used to estimate the SRSs prediction capability compared with the whole bladder.

Results: A local dose-effect relationship was found in the bladder and the urethra. SRSs were identified for five symptoms: acute incontinence in the urethra, acute retention in the bladder trigone, late retention and dysuria in the posterior part of the bladder, and late hematuria in the superior part of the bladder, with significant dose differences between patients with and without toxicity ranging from 1.2 to $9.3 \mathrm{~Gy}$. The doses to the SRSs were significantly predictive of toxicity, with maximum areas under the ROC curve (AUC) of 0.73 for acute incontinence, 0.62 for acute retention, 0.70 for late retention, 0.81 for late dysuria and 0.67 for late hematuria. The bladder DVH was predictive only for late retention, dysuria and hematuria (AUC $=0.65-0.72$ ). 
Conclusions: The dose delivered to the urethra, the posterior and superior part of the bladder was predictive of acute incontinence and retention and of late retention, dysuria and hematuria. The dose to the whole bladder was moderately predictive.

Keywords: prostate cancer, radiotherapy, urinary toxicity, voxel-wise analysis 


\section{INTRODUCTION}

Genitourinary (GU) toxicity following prostate radiotherapy (RT) is a common and limiting side effect. Urinary side effects are not fully understood, in particular how they relate to underlying dose-volume characteristics. Although patient-based risk factors of GU toxicity have been identified (1-5), the dosevolume response remains elusive. However, increasing evidence has recently arisen showing that localized anatomical subregions of the bladder are correlated with acute and late urinary toxicities (68) . Therefore, there is a clear need to exploit the rich information of the planning three-dimensional (3D) dose distribution, together with the individual's anatomy, to accurately identify subregions of the bladder/urethra at high risk of damage for toxicity prediction and more accurate organ-dose constraint definition. To this end, voxel-based methods for dose distribution analysis via dose-volume maps (DVMs) may help unravel the complexity of toxicity and local dose-volume relationships by identifying simultaneous involvement of different radiosensitive structures (9-11). The DVM approach is based on nonrigid registration to align patient anatomies and map dose distributions to a single reference (12). A subsequent voxel-wise statistical analysis is performed to test for local dose differences between patients with/without toxicity. Hence, organ subregions are computed as the clusters of voxels within the organ, where significant dose differences have been found. This methodology has been recently applied to identify regional dose differences in the rectum for gastrointestinal toxicity in prostate cancer $(9,10)$, in the heart and lung for corresponding toxicities in thoracic cancer $(13,14)$, and in the cricopharyngeus muscle and cervical esophagus for dysphagia in head and neck cancer (15). To our knowledge, a 3D voxel-based approach has never been applied to explore dosimetric patterns associated with urinary toxicity.

The objective of this study was to identify bladder and urethra subregions associated with urinary toxicity after prostate cancer RT. The proposed framework combines urethra segmentation, an accurate anatomical nonrigid registration approach for mapping the population 3D dose distributions to a single coordinate system, a voxel-wise analysis with respect to toxicity in the common space, and a further subregional analysis in the individual's space. 


\section{Materials AND Methods}

\section{Population data set, treatment, and urinary toxicity}

The study included a total of 272 patients with localized prostate cancer treated with intensitymodulated radiation therapy/image-guided radiation therapy (IMRT/IGRT) between May 2008 and July 2018 within two multicentric prospective phase III trials (STIC-IGRT) (16) and (PROFIT, normofractionated arm) (17). The mean age of the patients was 70 years (range: 52-81). The target volume included the prostate and seminal vesicles (SV), avoiding the pelvic lymph nodes. Target volume and organs at risks (bladder, rectum, and femoral heads) were delineated on computed tomography (CT) slices according to the GETUG and PROFIT recommendations. IMRT combined with IGRT was used to deliver a total dose of 78 Gy (in case of cone-beam CT [CBCT]) or 80 Gy (in case of fiducials) to the prostate over 8 weeks and 46 Gy to the SV over 4.6 weeks, at 2 Gy/fraction. Bladder dose-volume histograms (DVHs) were compiled either with GETUG recommendations as maximum dose $<80$ Gy or with PROFIT recommendations as $50 \%$ and $70 \%$ of the bladder wall receiving less than 53 Gy and 71 Gy, respectively. Patient and treatment characteristics are described in Table A1 (Supplementary material). All the patients provided informed consent. The trials were approved by the XXX (BLIND) Institutional Review Board and are registered in ClinicalTrials.gov (NCTXXX (BLIND) for the STIC-IGRT trial, NCTXXX (BLIND) for the PROFIT trial).

The median follow-up was 50 months (range: 6-102 months). Acute ( $\leq 3$ months from RT start date) and late (> 3months) urinary toxicity was scored using the CTCAE v.3.0 (210 patients from the STICIGRT trial) or the RTOG (62 patients from the PROFIT trial) radiation morbidity-scoring schema. We considered 20 endpoints: 5 symptoms (incontinence, retention, dysuria, hematuria, frequency), for both grade $\geq 1$ and grade $\geq 2$, and for acute and late urinary toxicity. The 5-year toxicity rates were estimated using Kaplan-Meier (KM) analysis. Additionally, a competing risk (CR) analysis was performed to estimate the cumulative incidence of each late toxicity endpoint by considering any other late toxicity as competing event (18) (Appendix 1). Table A2 (Supplementary material) displays the number of events and rates of acute and 5 -year late urinary toxicity (grade $\geq 1$ and grade $\geq 2$ ) by 
symptom as calculated by KM and CR models. Among the 20 endpoints, 4 symptoms (acute grade $\geq 2$ incontinence and hematuria, late grade $\geq 2$ dysuria and hematuria) were excluded from the analysis due to the absence or very low number of events.

The workflow of the study is divided into 7 steps, as presented in Figure 1.

\section{Automatic segmentation of the urethra on planning CT in the whole population (step 1)}

The urethra was automatically segmented with MABUS, a Multi Atlas-Based approach, as described by Acosta et al. (19) (see Appendix 2).

Template selection, registration of population to the template, and dose propagation (steps 2, 3, and 4)

An average patient, close to the whole population in terms of prostate volume, bladder volume, and urethral length, was selected as a common coordinate system (CCS) for aligning the entire population (step 2). A customized algorithm was devised for non-rigidly registering the structural description of the organs (bladder, urethra, and prostate; step 3) (12). The whole method is described in Appendix 3 and illustrated in Figure A1 (Supplementary material). Overall, Euclidean distance maps were combined with Laplacian scalar fields to build a structural description of the pelvis. Two-step registration was then performed by customizing parameters within the Elastix framework (20), which produces a final 3D deformation field used to propagate doses and structures to the CCS.

Geometric and dosimetric scores were used to quantitatively assess the registration accuracy. Thus, the centerline distance (CLD) was used for the urethra alignment (19) and the Dice Similarity Coefficient (DSC) and the Modified Hausdorff Distance (MHD) (21) for the prostate and the bladder. In addition, the dose-organ overlap (DOO) was computed to evaluate the dose-warping accuracy as in (10). Finally, the 3D registration uncertainty was estimated by computing the standard deviation of 
the center of mass coordinates of rigidly registered bladder contours. Subsequently, dose distributions for each patient were smoothed accordingly with a Gaussian kernel with corresponding width, to overcome registration uncertainties.

\section{DVM construction and definition of symptom-related subregions (SRSs) in the common coordinate system (step 5)}

Once the 3D dose distributions are propagated to the CCS, voxel-wise analysis allowed the identification of SRSs by generating for each symptom a DVM representing the dose differences between patients with and without toxicity. Only accurately registered patient data were included. Unilateral Mann-Whitney $U$ tests were voxel-wise performed between the two populations for each endpoint. The alternative hypothesis was that patients with toxicity received a higher dose. This step resulted in 3D maps for both dose differences and $P$-values (threshold at $P=0.01$; Figure 1, step 5), thereby characterizing each subregion in terms of average dose difference. To cope with the multiple comparisons problem, arising when performing multiple statistical tests (voxel-wise), a nonparametric permutation test (22) was performed (1000 permutations), which allowed the description of the entire DVM with a single adjusted $P$-value (Appendix 4). The resulting subregions were compared with those generated by the voxel-wise Mann-Whitney $U$ test (uncorrected). The overlapping regions (Mann Whitney $U$ test region and permutation test region) were finally considered in order to define the SRSs. The absolute volume (in cc) of each subregion in the CCS was also calculated.

\section{Inverse mapping of SRSs to the native patient space and toxicity prediction (steps 6 and 7)}

All segmented SRSs were propagated from the CCS back to each individual native space by applying the inverse of the previously computed 3D deformation field (step 6). Subsequently, DVHs and mean doses were computed for the SRSs, the urethra and the bladder and compared across the patients with and without toxicity. Unilateral Mann-Whitney $U$ test was used to compare the mean dose and each 
DVH bin between the two groups of patients. The prediction capability of the dose (mean and bin-wise) in the identified SRSs and the bladder was evaluated for the corresponding toxicity endpoints in the native space of the patients. Additionally, we tested if the doses in the SRSs by symptom identified for the grade $\geq 1$ toxicity endpoints were also predictive for grade $\geq 2$ toxicities, by symptom. For acute toxicities, the predictive performances of the SRSs and the bladder were estimated and compared using the logistic regression test. The $\mathrm{chi}^{2}$ test, was used to evaluate the association between categorical variables and acute toxicity. For continuous variables, the Man-Whitney U test was used. For late toxicities, the Cox proportional hazard model was used to compute risk estimates. The prediction capability of the Cox model at 5-years was evaluated with the area under the timedependent ROC curve (t-AUC), based on the approach proposed by Chambless and Diao (23), which accounts for censoring in survival analysis (also defined as cumulative/dynamic AUC). The AUC and t-

AUC and 95\% confidence intervals (CI) were computed using 1000 bootstrap replicates. The impact of patient and treatment characteristics on each toxicity endpoint was also assessed in a univariate analysis. Significant dosimetric and clinical parameters found in univariate analysis $(\mathrm{p} \leq 0.05)$, were included in multivariate logistic and Cox regression models for assessing acute and late toxicities, respectively. In a secondary analysis, while accounting for the presence of competing risks, the FineGray subdistribution hazard model was used to estimate the effect of covariates on the subdistribution hazard function of each event of interest in both univariate and multivariate analyses (24).

Statistical analysis and graphics were performed in Python and R.

\section{RESUlts}

\section{Accuracy of spatial normalization}

In total, 18 patients out of 272 were excluded from the study because of either low prostate $(\leq 0.88 ; n=$ 6) or bladder $(\leq 0.85 ; n=12)$ Dice scores. The cut-off points were empirically chosen after visual inspection of the registered images, considering both organ overlap and urethra alignment. For the remaining 254 patients, the computed mean values and standard deviations of DSC, MHD, and DOO 
scores for the prostate and the bladder and the CLD score for the urethra are reported in Table A3 (Supplementary material). The standard deviations of center of mass coordinates after the non-rigid registration were $2.4 \mathrm{~mm}$ in the left-right, $5.3 \mathrm{~mm}$ in the anterior-posterior and $6.2 \mathrm{~mm}$ in the cranialcaudal direction.

\section{Identification of the symptom-related subregions (SRSs) with significant dose differences between patients with/without toxicity, in the template (CCS)}

Figure 2 shows the SRSs where statistically significant dose differences between patients with/without toxicity appear in the common coordinate system (CCS). Figure 3 and the additional video show a 3D representation of the SRSs. SRSs were identified for a total of 5 grade $\geq 1$ symptoms: in the prostatic urethra for acute incontinence, in the bladder trigone for acute retention, late retention and in the posterior part of the bladder for dysuria, and in the superior part of the bladder for late hematuria. These volumes ranged from 2.4 to $16.9 \mathrm{cc}$ (Figure 2).

The dose differences across the patients (with and without toxicity) in acute and late retention SRSs were not statistically significant for grade $\geq 2$ toxicity. For the remaining SRSs, namely acute incontinence, late hematuria and late dysuria, the low number of grade $\geq 2$ events did not allow to perform a statistical analysis

\section{Dose differences between patients with/without toxicity in the native patient space}

The averaged dose differences between patients with/without toxicity in the five SRSs, in the whole bladder and the prostatic urethra after propagation of the SRS from the template to the native space, are reported in Table 1. These differences, ranging from 1.2 to $9.3 \mathrm{~Gy}$, were significant for the five SRSs $(\mathrm{p}<0.01)$. For the whole bladder, the dose differences were significant for acute retention (4.4 Gy), late retention (4.4 Gy) and late dysuria (9.3 Gy). A near-significant dose difference ( $p \leq 0.07)$ was found in the prostatic urethra for acute and late grade $\geq 2$ incontinence ( $\geq 0.8 \mathrm{~Gy}$ ). Figure 4 depicts the DVHs of 
the SRSs and the whole bladder for the two groups of patients. The dose bins with significant differences among the two populations are identified.

The code and template for generating the SRSs for an external patient are available as supplementary material.

\section{Predictive capabilities of the SRSs and whole bladder DVHs in the native spaces}

The predictive capabilities of the mean dose and the DVHs for the five SRSs and the whole bladder are shown in Table 2 (using the Cox model for late toxicity). The dose bins with the highest significant AUC/ t-AUC are reported. The AUC calculated from the doses in the SRSs ranged from 0.62 to 0.81 for the five symptoms (grade $\geq 1$ acute incontinence and retention, and late dysuria and retention), while the doses to the whole bladder were predictive only for late dysuria (highest AUC $=0.75$ ) and late retention (highest AUC=0.71). The results were confirmed when using the Fine-Gray model for late toxicity, except for hematuria (Table A4).

The doses delivered in the SRSs were not predictive of grade $\geq 2$ toxicity.

\section{Clinical parameters associated with urinary toxicity}

Table A5 shows the distribution of patient and treatment characteristics between the two groups (patients with/without acute toxicity). The results of univariate analysis testing the impact of patient and treatment parameters on acute toxicity are reported in Table A6; likewise for late toxicity using the Cox model in Table A7 and using the Fine Gray model in Table A8.

Table A9 presents the significant dosimetric and/or clinical parameters parameters impacting on acute and late urinary toxicity in multivariate analysis. The dosimetric impact of the doses to the SRSs is confirmed. Mean dose to the SRS combined to baseline retention symptoms increased slightly the late retention prediction capability $(\mathrm{AUC}=0.74)$, compared to mean dose to the SRS only (AUC=0.71). 


\section{DISCUSSION}

A robust nonrigid registration strategy, coupled with voxel-wise comparisons, allowed us to investigate the local dose-effect relationship for acute and 5-years late urinary toxicity after prostate cancer RT. The dose on five SRSs located in the urethra and the bladder were identified as good predictors for five grade $\geq 1$ symptoms (acute incontinence and retention, late retention, dysuria and hematuria). The predictive capabilities of these SRSs outperformed the predictive capabilities of the whole bladder. To our knowledge, this is the first study to explicitly correlate the dose to specific subregions of the bladder and the urethra with urinary toxicity, within a voxel-based framework. Given that the data were collected from clinical trials, these results may be generalized to a large part of the patient population.

Although the dose effect has been clearly demonstrated for rectal toxicity, its relationship with urinary toxicity has not been fully unveiled $(25,26)$. Indeed, urinary symptoms are multifactorial and depend on patient-specific clinical parameters $(3-5,27-30)$, individual biological patterns $(3,31-33)$ and dosimetric parameters. Moreover, urinary toxicity events may occur late after RT, in contrast to late gastro-intestinal toxicity which generally reaches a plateau after 3 years, suggesting that a longer follow-up is required to properly estimate late urinary toxicity (30).

An increasing number of studies have investigated the dose-effect relationship with respect to GU toxicity. Historically, the whole bladder's DVH has been used in an attempt to explain radiationinduced toxicities. Although some studies found a relationship between urinary toxicity $(7,27,34-37)$, others did not find any significant correlation $(38,39)$ This lack of homogeneity in the reported relationship between urinary toxicity and bladder dose can be attributed to several factors (25). The planning dose may not be representative of the actual dose delivered because of the high variability in the interfraction bladder volume $(40,41)$. In this context, dose-surface histograms (DSH) have been shown to be better dosimetry surrogates than DVHs for acute urinary toxicity (42). DSH appears also 
stable with respect to bladder interfraction variations in the high-dose region (43). However, the limited prediction capability of whole-bladder DVHs/DSHs may be related to the lack of spatial information. Indeed, increasing evidence suggests that different bladder subregions might be linked with different urinary functions $(6,7,27,44,45)$. Historically, the simplest approach was to manually delineate bladder subvolumes (7) or use a parameterized representation of the dose to the bladder (27). Despite the limitations of these studies in terms of spatial accuracy, associations between the trigone dose and late urinary side effects have been reported, thus raising questions concerning the involvement of specific subregions in urinary toxicity.

Beyond the DVHs, 2D pixel-wise approaches based on dose-surface maps (DSMs) and 3D voxel-based methods via DVMs have been recently proposed to describe the local dose-effect relationship. The DSMs, representing a mapping of the dose delivered to the organ's surface, are constructed by virtually unfolding the organ in a slice wise manner. They have been used to explain rectal toxicity by extracting dose features (46-48) and urinary toxicity by comparing pixel wise dose descriptors $(6,8,44)$. In total, three studies used DSMs to investigate acute $(8,44)$ and late urinary toxicity $(6)$. Concerning acute toxicity analyzed by DSMs, urinary frequency/urgency was significantly related to the dose at 5-12 $\mathrm{mm}$ posterior to the bladder base, corresponding to trigone (44). Concerning late toxicity, another DSM analysis (6) found incontinence to be related to the region lateral to trigone. They also reported that the dose to the anterior-inferior and posterior-superior bladder surface was associated with worsening of symptoms as measured by IPSS, dysuria, and hematuria.

Nevertheless, DSMs remain a 2D representation of the dose to the organs' surface and are built for each organ depending on its particular geometry. On the contrary, DVMs reflect the 3D dose delivered to the entire volume without any prior assumptions on anatomical regions. Our study is the first to explicitly correlate the 3D dose to the urethra with urinary toxicity following external beam radiation therapy. Acute and late retention SRSs were found in the trigone and posterior part of the bladder. Indeed, retention can be due to a reduced bladder contractility (detrusor muscle). Relationship between the dose to the bladder trigone or high bladder-dose and obstructive side effects, has also been reported in the literature $(7,27,49)$. Dysuria (painful urination or difficulty urinating) SRS is also 
mainly located in the posterior part of the bladder, partially in the trigone. Surprisingly, a SRS for hematuria was found superiorly, at the bladder dome (a region which receives a relatively low dose, < $20 \mathrm{~Gy})$, while bleeding classically related to high dose $(3,49,50)$, and telangiectasia are mainly observed in the bladder neck/trigone at the cystoscopy. Our results for dysuria and hematuria are however in line with another DSM study (6). Incontinence appears related to the dose delivered to the prostatic urethra. Indeed, incontinence may result from malfunctioning of the urethral sphincter (51). A dosimetric association with urinary frequency could not be demonstrated in the present study, although this symptom was strongly predicted by the baseline frequency (Table A9). Furthermore, any SRS could be located in the superior-anterior part of the dome as this part of the bladder received a dose close to zero (Figure 2).

One of the major advantages of the voxel-wise analysis is that the whole 3D volume can be explored and compared without any prior knowledge of regions correlating with toxicity. However, our approach relies on a multi-organ deformable image registration, which appears particularly challenging given the high interindividual variability. Conventional interindividual CT registration methods are not accurate enough for reliable anatomical mapping because of the low soft-tissue contrast. To cope with this issue, we combined a structural description of the pelvic region using the contours obtained within the clinical protocol (described in Appendix 2). Each step was thoroughly validated, including the automatic segmentation of the urethra, thus increasing the reliability of dose mapping. We propagated the SRSs found in the template back to each individual's space and, as such RT planning may be modified to spare specific subregions. The feasibility of reducing the dose in the SRSs, while preserving the dose to the PTV, still requires demonstration. This goal seems achievable when dealing with SRSs distant from the PTV (such as hematuria SRS) but much more difficult when SRSs are located inside or close to the PTV (such as incontinence SRS).

Our study presents some limitations. The results must be carefully interpreted, as a correlation between local dose and toxicity does not necessarily mean causality, especially given the paucity of events for certain endpoints. Second, we failed to find a spatial dose-effect relationship for some symptoms, in particular for grade $\geq 2$ toxicity. Although this may imply the absence of specific SRSs for 
these symptoms, it can also be due to the limited follow-up or to the patient-specific parameters that affect toxicity and were not taken into account or to the limited follow-up. Furthermore, the predictive performance of our model has been internally validated (via bootstrapping) on the same subjects used to construct the model. External cohorts are therefore required to validate the results. Finally, statistical analyses were performed on the planning dose distribution, which can differ from the actual delivered dose $(40,41)$. Daily 3D imaging, such as CBCT, could be used to compute cumulated doses, thereby confirming some of the SRSs' implication in toxicity (52).

\section{ConClusion}

A voxel-wise analysis allowed the identification of urethro-vesical SRSs, whose irradiation appears highly correlated to specific urinary side effects after prostate cancer RT. The dose received by these SRSs was more predictive than the dose to the whole bladder. 


\section{REFERENCES}

1. Rancati T, Cozzarini C, Improta I, et al. Multivariable models predicting specific patient-reported acute urinary symptoms after radiation therapy for prostate cancer: Results of a cohort study. Int. J. Radiat. Oncol. Biol. Phys. 2015;93:E240-E241.

2. Ghadjar P, Jackson A, Spratt DE, et al. Patterns and predictors of amelioration of genitourinary toxicity after high-dose intensity-modulated radiation therapy for localized prostate cancer: Implications for defining postradiotherapy urinary toxicity. Eur. Urol. 2013;64:931-938.

3. De Langhe S, De Meerleer G, De Ruyck K, et al. Integrated models for the prediction of late genitourinary complaints after high-dose intensity modulated radiotherapy for prostate cancer: Making informed decisions. Radiother. Oncol. 2014;112:95-99.

4. Yahya N, Ebert MA, Bulsara M, et al. Dosimetry, clinical factors and medication intake influencing urinary symptoms after prostate radiotherapy: An analysis of data from the RADAR prostate radiotherapy trial. Radiother. Oncol. 2015;116:112-118.

5. Barnett GC, De Meerleer G, Gulliford SL, et al. The Impact of Clinical Factors on the Development of Late Radiation Toxicity: Results from the Medical Research Council RT01 Trial (ISRCTN47772397). Clin. Oncol. 2011;23:613-624.

6. Yahya N, Ebert MA, House MJ, et al. Modeling Urinary Dysfunction After External Beam Radiation Therapy of the Prostate Using Bladder Dose-Surface Maps: Evidence of Spatially Variable Response of the Bladder Surface. Int. J. Radiat. Oncol. 2017;97:420-426.

7. Ghadjar P, Zelefsky MJ, Spratt DE, et al. Impact of dose to the bladder trigone on long-term urinary function after high-dose intensity modulated radiation therapy for localized prostate cancer. Int. J. Radiat. Oncol. Biol. Phys. 2014;88:339-344.

8. Improta I, Palorini F, Cozzarini C, et al. Bladder spatial-dose descriptors correlate with acute urinary toxicity after radiation therapy for prostate cancer. Phys. Medica. 2016;32:1681-1689.

9. Acosta O, Drean G, Ospina JD, et al. Voxel-based population analysis for correlating local dose and rectal toxicity in prostate cancer radiotherapy. Phys. Med. Biol. 2013;58:2581-95. 
10. Dréan G, Acosta O, Ospina JD, et al. Identification of a rectal subregion highly predictive of rectal bleeding in prostate cancer IMRT. Radiother. Oncol. 2016;119:388-97.

11. Acosta O, De Crevoisier R. Beyond DVH: 2D/3D based dose comparison to assess predictors of toxicity. In: Fiorino C, Tiziana R, eds. Modelling Radiotherapy Side Effects Practical Applications for Planning Optimization. CRC Pr I Llc; 2018.

12. Dréan G, Acosta O, Lafond C, et al. Interindividual registration and dose mapping for voxelwise population analysis of rectal toxicity in prostate cancer radiotherapy. Med. Phys. 2016;43:2721-2730. 13. Palma G, Monti S, Avino VD, et al. A Voxel-Based Approach to Explore Local Dose Differences Associated With Radiation-Induced Lung Damage. Radiat. Oncol. Biol. 2016;96:127-133.

14. McWilliam A, Kennedy J, Hodgson C, et al. Radiation dose to heart base linked with poorer survival in lung cancer patients. Eur. J. Cancer. 2017;85:106-113.

15. Monti S, Palma G, D’Avino V, et al. Voxel-based analysis unveils regional dose differences associated with radiation-induced morbidity in head and neck cancer patients. Sci. Rep. 2017;7:7220.

16. de Crevoisier R, Bayar MA, Pommier P, et al. Daily Versus Weekly Prostate Cancer Image Guided Radiation Therapy: Phase 3 Multicenter Randomized Trial. Int. J. Radiat. Oncol. 2018;102:1420-1429. 17. Catton CN, Lukka H, Gu C-S, et al. Randomized Trial of a Hypofractionated Radiation Regimen for the Treatment of Localized Prostate Cancer. J. Clin. Oncol. 2017;35:1884-1890.

18. Ravi Varadhan COWJBSAWWDSCB. Evaluating Health Outcomes in the Presence of Competing Risks: A Review of Statistical Methods and Clinical Applications. Med. Care. 2010;48:S96-S105. 19. Acosta O, Mylona E, Le Dain M, et al. Multi-atlas-based segmentation of prostatic urethra from planning CT imaging to quantify dose distribution in prostate cancer radiotherapy. Radiother. Oncol. 2017;125:492-499.

20. Klein S, Staring M, Murphy K, et al. elastix: A Toolbox for Intensity-Based Medical Image Registration. IEEE Trans. Med. Imaging. 2010;29:196-205.

21. Dubuisson M-P, Jain AK. A modified Hausdorff distance for object matching. In: Proceedings of 12th International Conference on Pattern Recognition.Vol 1. IEEE Comput. Soc. Press;566-568.

22. Chen C, Witte M, Heemsbergen W, et al. Multiple comparisons permutation test for image based data mining in radiotherapy. Radiat. Oncol. 2013;8:293. 
23. Chambless LE, Diao G. Estimation of time-dependent area under the ROC curve for long-term risk prediction. Stat. Med. 2006;25:3474-3486.

24. Fine JP, Gray RJ. A Proportional Hazards Model for the Subdistribution of a Competing Risk. J. Am. Stat. Assoc. 1999;94:496-509.

25. Rosewall T, Catton C, Currie G, et al. The relationship between external beam radiotherapy dose and chronic urinary dysfunction - A methodological critique. Radiother. Oncol. 2010;97:40-47.

26. Landoni V, Fiorino C, Cozzarini C, et al. Predicting toxicity in radiotherapy for prostate cancer. Phys. Medica. 2016;32:521-532.

27. Heemsbergen WD, Al-Mamgani A, Witte MG, et al. Urinary obstruction in prostate cancer patients from the dutch trial (68 Gy vs. 78 Gy): Relationships with local dose, acute effects, and baseline characteristics. Int. J. Radiat. Oncol. Biol. Phys. 2010;78:19-25.

28. Cozzarini C, Rancati T, Carillo V, et al. Multi-variable models predicting specific patient-reported acute urinary symptoms after radiotherapy for prostate cancer: Ad interim results of a cohort study. Eur. Urol. Suppl. 2014;13:114-115.

29. Ahmed AA, Egleston B, Alcantara P, et al. A novel method for predicting late genitourinary toxicity after prostate radiation therapy and the need for age-based risk-adapted dose constraints. Int. J. Radiat. Oncol. Biol. Phys. 2013;86:709-715.

30. Mathieu R, Arango JDO, Beckendorf V, et al. Nomograms to predict late urinary toxicity after prostate cancer radiotherapy. World J. Urol. 2014;32:743-751.

31. Barnett GC, Coles CE, Elliott RM, et al. Independent validation of genes and polymorphisms reported to be associated with radiation toxicity: a prospective analysis study. Lancet Oncol. 2012;13:65-77.

32. Smith JJ, Wasserman I, Milgrom SA, et al. Single Nucleotide Polymorphism TGFß1 R25P Correlates with Acute Toxicity during Neoadjuvant Chemoradiotherapy in Rectal Cancer Patients. Int. J. Radiat. Oncol. Biol. Phys. 2017;97:924-930.

33. Someya M, Hori M, Gocho T, et al. Prediction of acute gastrointestinal and genitourinary radiation toxicity in prostate cancer patients using lymphocyte microRNA. Jpn. J. Clin. Oncol. 2018;48:167-174. 34. Harsolia A, Vargas C, Yan D, et al. Predictors for Chronic Urinary Toxicity After the Treatment of 
Prostate Cancer With Adaptive Three-Dimensional Conformal Radiotherapy: Dose-Volume Analysis of a Phase II Dose-Escalation Study. Int. J. Radiat. Oncol. Biol. Phys. 2007;69:1100-1109.

35. Pederson AW, Fricano J, Correa D, et al. Late toxicity after intensity-modulated radiation therapy for localized prostate cancer: An exploration of dose-volume histogram parameters to limit genitourinary and gastrointestinal toxicity. Int. J. Radiat. Oncol. Biol. Phys. 2012;82:235-241.

36. Palorini F, Rancati T, Cozzarini C, et al. Multi-variable models of large International Prostate Symptom Score worsening at the end of therapy in prostate cancer radiotherapy. Radiother. Oncol. 2016;118:92-98.

37. Thor M, Olsson C, Oh JH, et al. Urinary bladder dose-response relationships for patient-reported genitourinary morbidity domains following prostate cancer radiotherapy. Radiother. Oncol. 2016;119:117-122.

38. Boersma LJ, Van Den Brink M, Bruce AM, et al. Estimation of the incidence of late bladder and rectum complications after high-dose (70-78 Gy) conformal radiotherapy for prostate cancer, using dose-volume histograms. Int. J. Radiat. Oncol. Biol. Phys. 1998;41:83-92.

39. Storey MR, Pollack A, Zagars G, et al. Complications from radiotherapy dose escalation in prostate cancer: preliminary results of a randomized trial. Int. J. Radiat. Oncol. 2000;48:635-642.

40. Nassef M, Simon A, Cazoulat G, et al. Quantification of dose uncertainties in cumulated dose estimation compared to planned dose in prostate IMRT. Radiother. Oncol. 2016;119:129-136.

41. Pearson D, Gill SK, Campbell N, et al. Dosimetric and volumetric changes in the rectum and bladder in patients receiving CBCT-guided prostate IMRT: analysis based on daily CBCT dose calculation. J. Appl. Clin. Med. Phys. 2016;17:107-117.

42. Carillo V, Cozzarini C, Chietera A, et al. Correlation between surrogates of bladder dosimetry and dose-volume histograms of the bladder wall defined on MRI in prostate cancer radiotherapy. Radiother. Oncol. 2012;105:180-183.

43. Palorini F, Botti A, Carillo V, et al. Bladder dose-surface maps and urinary toxicity: Robustness with respect to motion in assessing local dose effects. Phys. Medica. 2016;32:506-511.

44. Palorini F, Cozzarini C, Gianolini S, et al. First application of a pixel-wise analysis on bladder dosesurface maps in prostate cancer radiotherapy. Radiother. Oncol. 2016;119:123-128. 
45. Viswanathan AN, Yorke ED, Marks LB, et al. Radiation Dose-Volume Effects of the Urinary Bladder. Int. J. Radiat. Oncol. Biol. Phys. 2010;76:116-122.

46. Wortel RC, Witte MG, van der Heide UA, et al. Dose-surface maps identifying local dose-effects for acute gastrointestinal toxicity after radiotherapy for prostate cancer. Radiother. Oncol. 2015;117:515520.

47. Buettner F, Gulliford SL, Webb S, et al. Using dose-surface maps to predict radiation-induced rectal bleeding: a neural network approach. Phys. Med. Biol. 2009;54:5139-5153.

48. Buettner F, Gulliford SL, Webb S, et al. Modeling late rectal toxicities based on a parameterized representation of the 3D dose distribution. Phys. Med. Biol. 2011;56:2103-2118.

49. Olsson CE, Jackson A, Deasy JO, et al. A Systematic Post-QUANTEC Review of Tolerance Doses for Late Toxicity After Prostate Cancer Radiation Therapy. Int. J. Radiat. Oncol. 2018;102:1514-1532.

50. Yahya N, Ebert MA, Bulsara M, et al. Urinary symptoms following external beam radiotherapy of the prostate: Dose-symptom correlates with multiple-event and event-count models. Radiother. Oncol. 2015.

51. Nitti VW. The prevalence of urinary incontinence. Rev. Urol. 2001;3 Suppl 1:S2-6.

52. Delobel J-B, Gnep K, Ospina JD, et al. Nomogram to predict rectal toxicity following prostate cancer radiotherapy. PLoS One. 2017;12:e0179845.

53. Koller MT, Raatz H, Steyerberg EW, et al. Competing risks and the clinical community: irrelevance or ignorance? Stat. Med. 2012;31:1089-1097.

54. Winkler AM, Ridgway GR, Webster MA, et al. Permutation inference for the general linear model. Neuroimage. 2014;92:381-397.

55. Scarpazza C, Tognin S, Frisciata S, et al. False positive rates in Voxel-based Morphometry studies of the human brain: Should we be worried? Neurosci. Biobehav. Rev. 2015;52:49-55.

56. Ashburner J, Friston KJ. Voxel-Based Morphometry-The Methods. Neuroimage. 2000;11:805-821. 57. Smith SM, Nichols TE. Threshold-free cluster enhancement: Addressing problems of smoothing, threshold dependence and localisation in cluster inference. Neuroimage. 2009;44:83-98. 


\section{Table and figure legends}

Table 1. Dose differences between patients with/without acute (A) and late (B) urinary toxicity in the whole bladder, the prostatic urethra and the symptom-related subregions (SRSs) in the native space of the patients

SRS: Symptom-related subregion; SD: Standard deviation, SE: Standard error; NS: not significant ( $p$ > $0.05)$

Each SRS represents the subregion for the corresponding symptom (when identified). The Wilcoxon test has been used to calculate the p-values. The doses are not given for grade $\geq 2$ acute incontinence and hematuria and late dysuria and hematuria due to the absence or very low number of events.

Table 2. Urinary toxicity prediction capability of the mean dose and the DVH for the whole bladder and the identified symptom-related subregions (SRSs) in the native space of the patients

SRS: Symptom-related subregion; OR: Odds ratio; HR: Hazard ratio; CI: Confidence interval; AUC: Area under the ROC curve; t-AUC: time-dependent area under the ROC curve; NS: not significant ( $p>0.05)$; *DVH bin with the highest AUC and range of bins with statistically significant $\mathrm{p}$ value $(\leq 0.05)$

Univariate analysis was performed using logistic regression for acute toxicity and Cox regression for late toxicity. The predictive performance for acute toxicity was estimated using the area under the ROC curve. The predictive performance for late toxicity was estimated at 5-years using the area under the time-dependent ROC curve (t-AUC). Doses were not found predictive for grade $\geq 2$ toxicity.

Figure 1. Workflow of the study in 7 steps 
Figure 2. Symptom-related subregions (SRSs) of statistically significant dose differences between patients with/without toxicity in the common coordinate system (CCS)

$\mathrm{A}=$ =anterior; $\mathrm{P}=$ posterior; $\mathrm{R}=$ right; $\mathrm{L}=\mathrm{left} ; \Delta$ : difference

Sagittal and coronal views of the template showing the identified SRSs for five symptoms (grade $\geq 1$ ), as defined with permutation tests (corrected p-value $<0.2$ ). On the left is shown the dose distribution to the bladder and the urethra for patients with/without toxicity. The dose displayed in each voxel corresponds to the mean dose of each population. The mean (standard deviation) dose received by the whole SRSs in each population is given in the figure. On the right is shown the mean dose difference to the bladder and the urethra (sagittal and coronal views). The white rectangle indicates the region of the template's trigone.

Figure 3. Identified symptom-related subregions (SRSs) in the in the common coordinate system (template)

$\mathrm{A}=$ anterior; $\mathrm{P}=$ posterior; $\mathrm{R}=$ right; $\mathrm{L}=\mathrm{left}$

The symptoms correspond to grade $\geq 1$ toxicity. The corresponding video shows a 3D representation of the SRSs.

Figure 4. Dose-volume histograms of patients with and without urinary toxicity for the whole bladder and the symptom-related subregions (SRSs) in the native space

The red and green curves represent the average DVHs of the patient with/without toxicity, respectively, in the SRSs (continuous lines) and in the whole bladder (dashed lines). The symptoms correspond to grade $\geq 1$ toxicity. The shadowed region indicates the dose bins where the dose for the group with toxicity is significantly higher than the group without toxicity (Man-Whitney $U$ test; $\mathrm{p} \leq$ 0.05). 


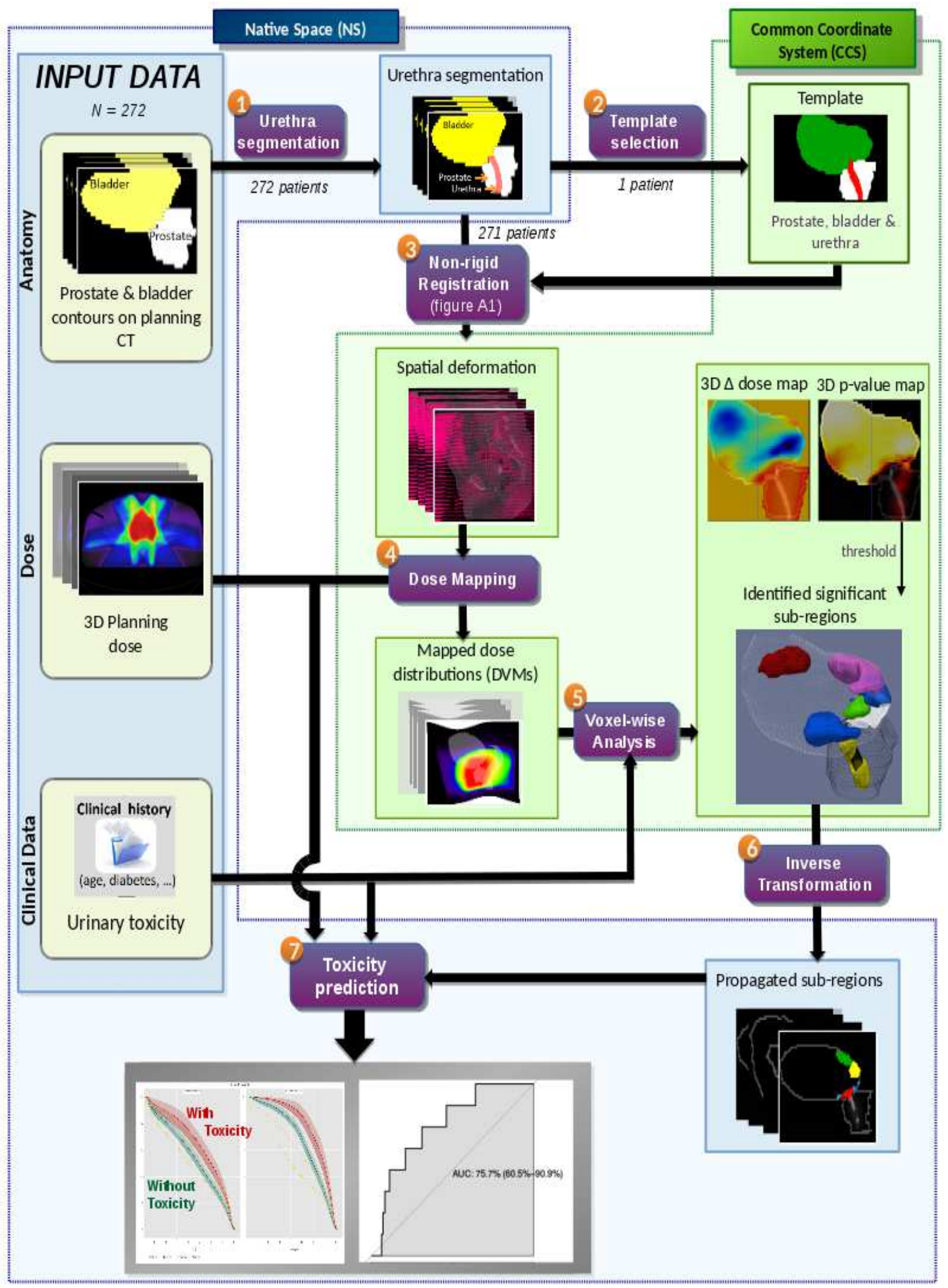

Figure 1. Workflow of the study in 7 steps 


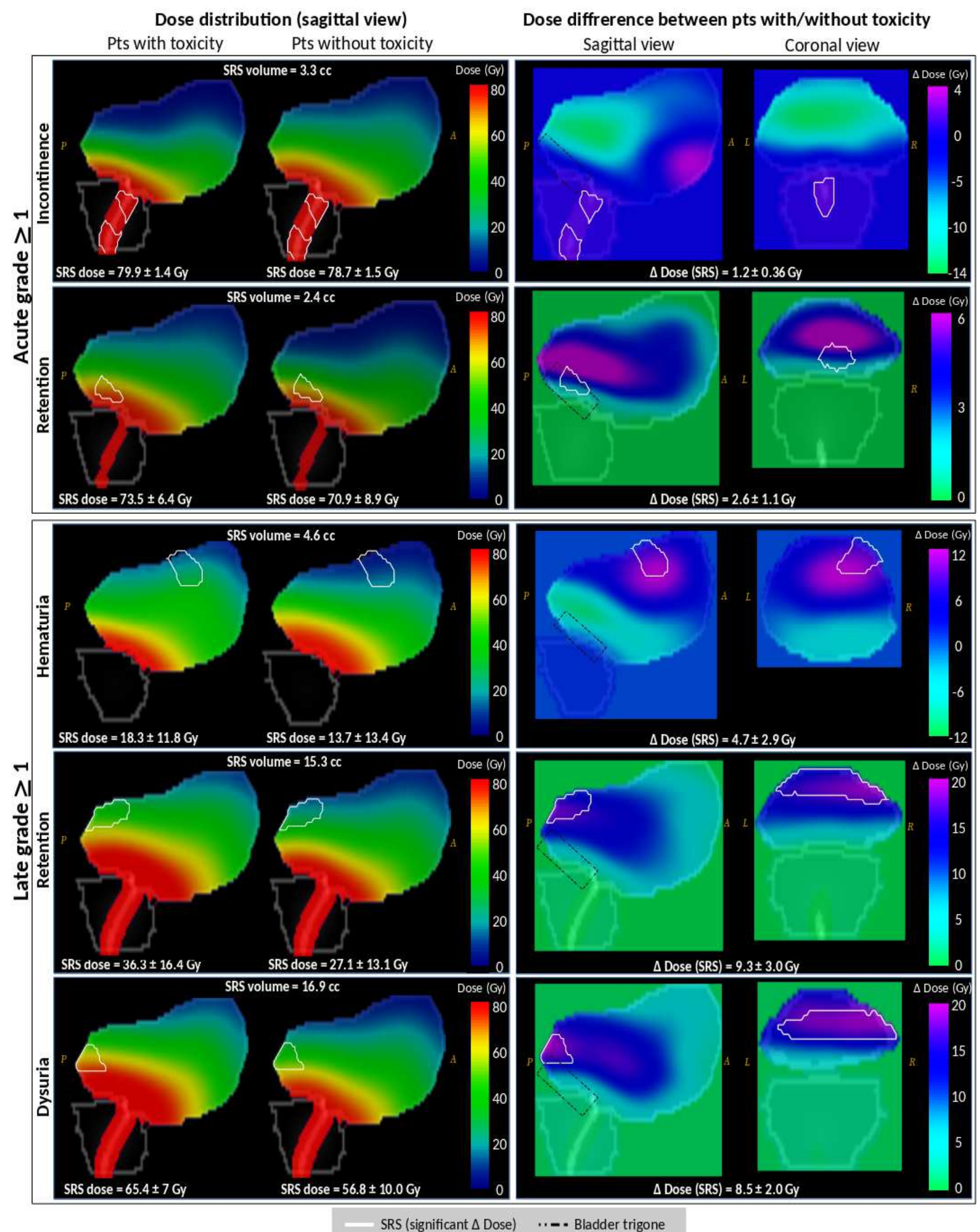

Figure 2. Symptom-related subregions (SRSs) of statistically significant dose differences between patients with/without toxicity in the common coordinate system (CCS)

$\mathrm{A}=$ anterior; $\mathrm{P}=$ posterior; $\mathrm{R}=$ right; $\mathrm{L}=\mathrm{left} ; \Delta$ : difference 
Sagittal and coronal views of the template showing the identified SRSs for five symptoms (grade $\geq$ 1 ), as defined with permutation tests (corrected p-value <0.2). On the left is shown the dose distribution to the bladder and the urethra for patients with/without toxicity. The dose displayed in each voxel corresponds to the mean dose of each population. The mean (standard deviation) dose received by the whole SRSs in each population is given in the figure. On the right is shown the mean dose difference to the bladder and the urethra (sagittal and coronal views). The white rectangle indicates the region of the template's trigone. 


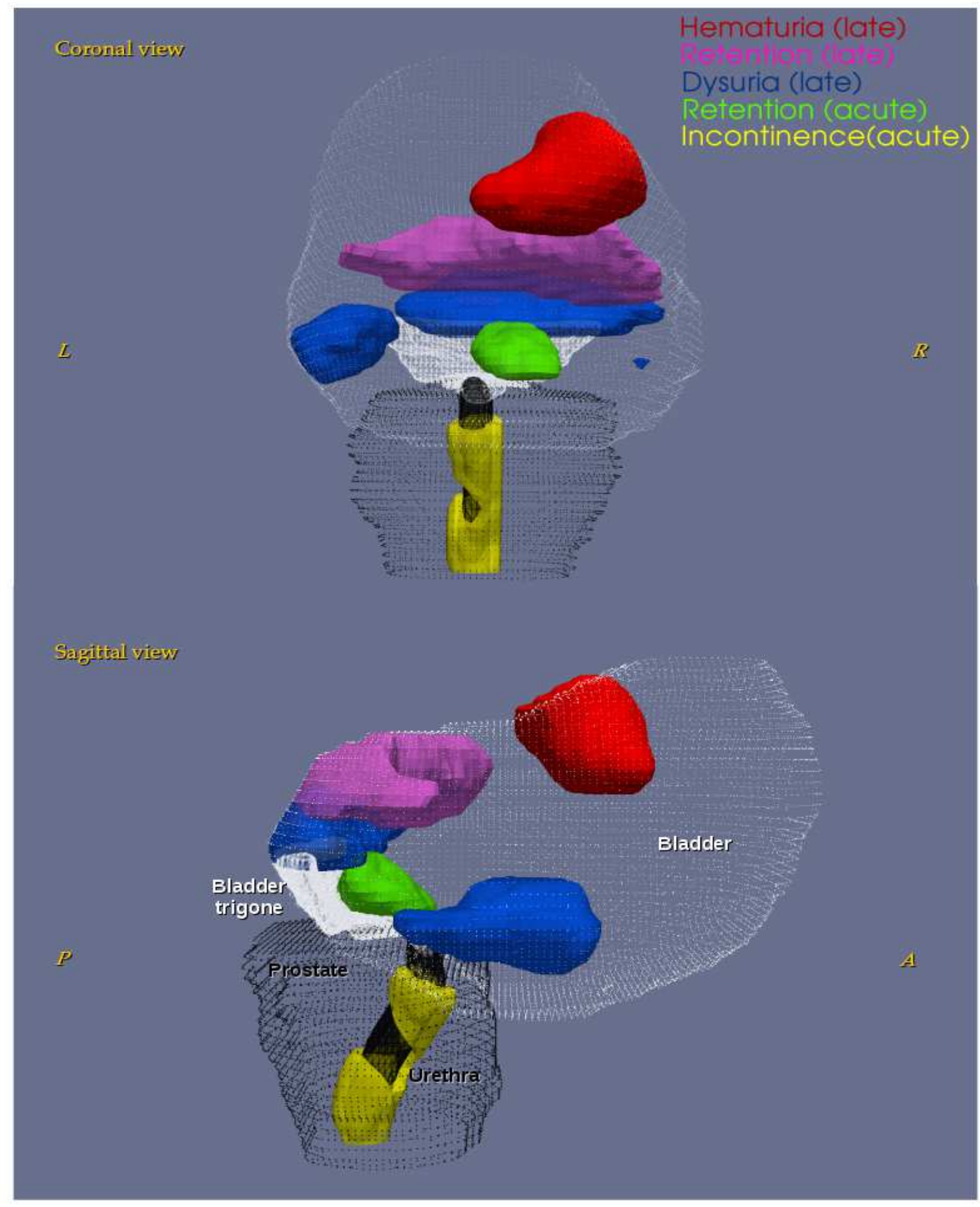

Figure 3. Identified symptom-related subregions (SRSs) in the in the common coordinate system (template)

$\mathrm{A}=$ anterior; $\mathrm{P}=$ posterior; $\mathrm{R}=$ right; $\mathrm{L}=\mathrm{left}$

The symptoms correspond to grade $\geq 1$ toxicity. The corresponding video shows a 3D representation of the SRSs. 

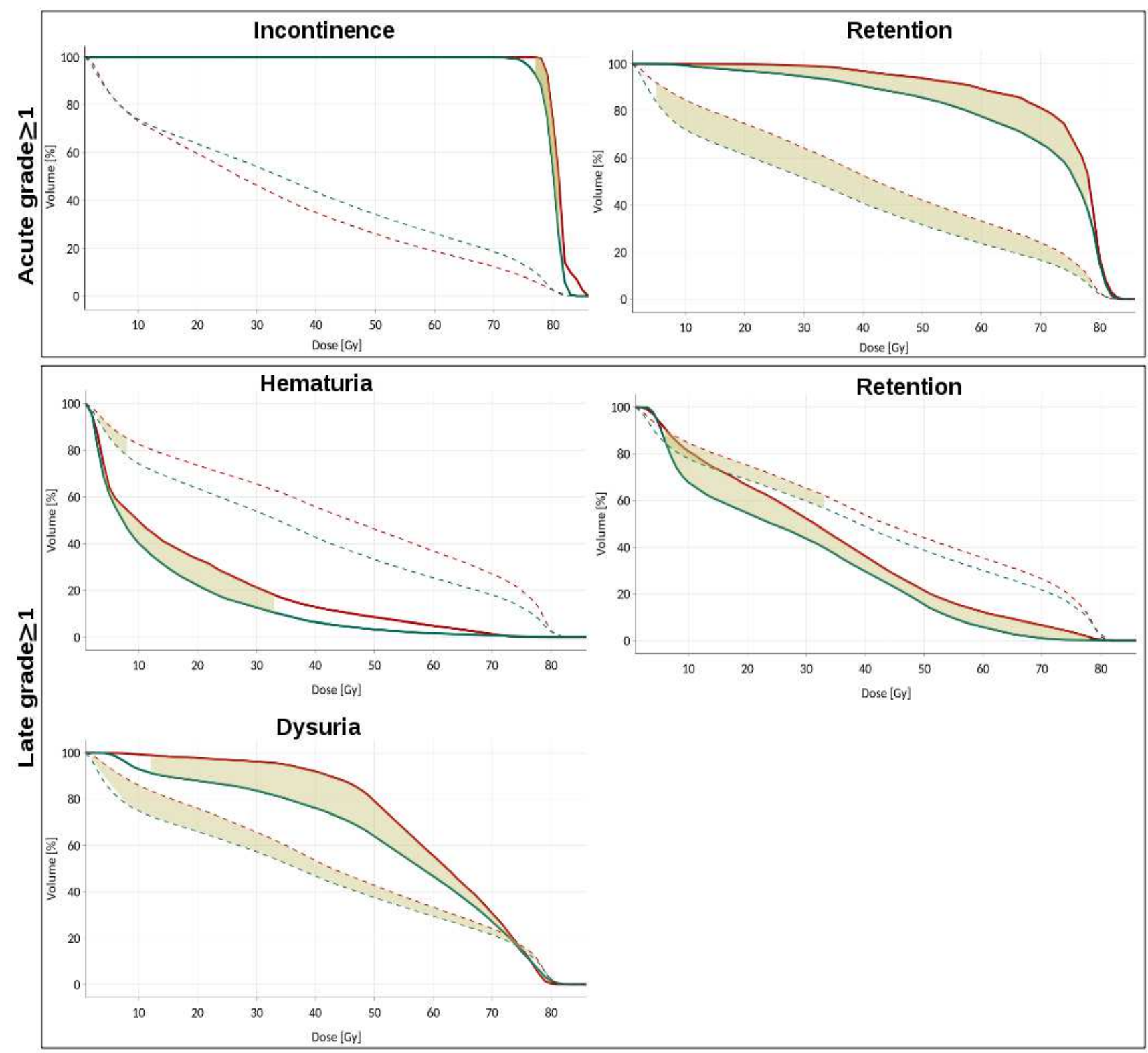

- SRS DVH of pts with toxicity _ _ SRS DVH of pts without toxicity $\quad$ - - Bladder DVH of pts with toxicity - - Bladder DVH of pts without toxicity Region of statistically significant dose difference (per bin, $\mathrm{p}<0.05$ )

Figure 4. Dose-volume histograms of patients with and without urinary toxicity for the whole bladder and the symptom-related subregions (SRSs) in the native space

The red and green curves represent the average DVHs of the patient with/without toxicity, respectively, in the SRSs (continuous lines) and in the whole bladder (dashed lines). The symptoms correspond to grade $\geq 1$ toxicity. The shadowed region indicates the dose bins where the dose for the group with toxicity is significantly higher than the group without toxicity (Man-Whitney U test; $\mathrm{p} \leq 0.05)$. 
Table 1. Dose differences between patients with/without acute (A) and late (B) urinary toxicity in the whole bladder, the prostatic urethra and the symptom-related subregions (SRSs) in the native space of the patients

\section{A: Acute urinary toxicity}

\begin{tabular}{|c|c|c|c|c|c|}
\hline \multicolumn{6}{|c|}{ Grade $\geq 1$} \\
\hline Endpoints & Regions & $\begin{array}{l}\text { Dose of pts with toxicity } \\
\text { (mean } \pm S D, G y)\end{array}$ & $\begin{array}{l}\text { Dose of pts without toxicity } \\
\text { (mean } \pm \text { SD , Gy) }\end{array}$ & $\begin{array}{l}\text { Dose difference } \\
\text { (mean } \pm \mathrm{SE}, \mathrm{Gy})\end{array}$ & p value \\
\hline \multirow{3}{*}{ Hematuria } & Whole bladder & $39.5 \pm 15.8$ & $35.3 \pm 14.4$ & $4.2 \pm 6.05$ & NS \\
\hline & Prostatic urethra & $77.9 \pm 0.4$ & $78.5 \pm 0.5$ & $-0.6 \pm 0.2$ & NS \\
\hline & SRS & - & - & - & - \\
\hline \multirow{3}{*}{ Dysuria } & Whole bladder & $37.5 \pm 15.4$ & $38.1 \pm 14.9$ & $-0.6 \pm 2.3$ & NS \\
\hline & Prostatic urethra & $78.3 \pm 0.5$ & $78.5 \pm 0.5$ & $-0.3 \pm 0.1$ & NS \\
\hline & SRS & - & - & - & - \\
\hline \multirow{3}{*}{ Retention } & Whole bladder & $40.4 \pm 14.6$ & $36 \pm 15.3$ & $4.4 \pm 2.3$ & 0.03 \\
\hline & Prostatic urethra & $78.4 \pm 0.3$ & $78.4 \pm 0.4$ & $-0.05 \pm 0.1$ & NS \\
\hline & SRS & $73.5 \pm 6.4$ & $70.9 \pm 8.9$ & $2.6 \pm 1.2$ & $<0.01$ \\
\hline \multirow{3}{*}{ Frequency } & Whole bladder & $35.6 \pm 14.8$ & $34.7 \pm 12.8$ & $0.9 \pm 2.1$ & NS \\
\hline & Prostatic urethra & $78.5 \pm 0.7$ & $78.5 \pm 0.7$ & 0 & NS \\
\hline & SRS & - & - & - & - \\
\hline \multirow{3}{*}{ Incontinence } & Whole bladder & $31.3 \pm 6.5$ & $35.7 \pm 14.7$ & $-4.4 \pm 1.9$ & NS \\
\hline & Prostatic urethra & $79.3 \pm 1.2$ & $78.5 \pm 0.9$ & $0.8 \pm 0.5$ & 0.06 \\
\hline & SRS & $79.9 \pm 1.4$ & $78.7 \pm 1.5$ & $1.2 \pm 0.3$ & $<0.01$ \\
\hline \multicolumn{6}{|c|}{ Grade $\geq 2$} \\
\hline \multirow{3}{*}{ Dysuria } & Whole bladder & $35.5 \pm 17.3$ & $38 \pm 15$ & $-2.5 \pm 4.9$ & NS \\
\hline & Prostatic urethra & $78.5 \pm 0.5$ & $78.2 \pm 0.4$ & $0.3 \pm 0.2$ & NS \\
\hline & SRS & - & - & - & - \\
\hline \multirow{3}{*}{ Retention } & Whole bladder & $38.8 \pm 15.8$ & $37.6 \pm 15$ & $1.2 \pm 2.9$ & NS \\
\hline & Prostatic urethra & $78.2 \pm 0.3$ & $78.5 \pm 0.4$ & $-0.3 \pm 0.1$ & NS \\
\hline & SRS & - & - & - & - \\
\hline \multirow{3}{*}{ Frequency } & Whole bladder & $36.6 \pm 14.7$ & $35 \pm 14.3$ & $1.6 \pm 2$ & NS \\
\hline & Prostatic urethra & $78.6 \pm 0.8$ & $78.4 \pm 0.7$ & $0.2 \pm 0.1$ & NS \\
\hline & SRS & - & - & - & - \\
\hline
\end{tabular}




\section{B. Late urinary toxicity (at 5 years)}

\begin{tabular}{|c|c|c|c|c|c|}
\hline \multicolumn{6}{|c|}{ Grade $\geq 1$} \\
\hline Endpoints & Regions & $\begin{array}{l}\text { Dose of pts with toxicity } \\
\text { (mean } \pm \text { SD , Gy) }\end{array}$ & $\begin{array}{l}\text { Dose of pts without toxicity } \\
\text { (mean }+ \text { SD , Gy) }\end{array}$ & $\begin{array}{l}\text { Dose difference } \\
\text { (mean } \pm \text { SE , Gy) }\end{array}$ & $p$ value \\
\hline \multirow{3}{*}{ Hematuria } & Whole bladder & $38.7 \pm 11.5$ & $39.8 \pm 13.4$ & $-1.1 \pm 2.8$ & NS \\
\hline & Prostatic urethra & $77.7 \pm 1.1$ & $78.1 \pm 0.6$ & $-0.4 \pm 0.7$ & NS \\
\hline & SRS & $18.3 \pm 11.8$ & $13.7 \pm 13.4$ & $4.69 \pm 2.9$ & 0.04 \\
\hline \multirow{3}{*}{ Dysuria } & Whole bladder & $50.1 \pm 10.1$ & $40.8 \pm 12.9$ & $9.3 \pm 2.8$ & $<0.01$ \\
\hline & Prostatic urethra & $78 \pm 0.3$ & $77.9 \pm 0.5$ & $0.1 \pm 0.4$ & NS \\
\hline & SRS & $65.4 \pm 7$ & $56.8 \pm 10.0$ & $8.5 \pm 2$ & $<0.01$ \\
\hline \multirow{3}{*}{ Retention } & Whole bladder & $46 \pm 13.9$ & $41.6 \pm 12.5$ & $4.4 \pm 2.7$ & 0.05 \\
\hline & Prostatic urethra & $78.1 \pm 0.4$ & $78 \pm 0.6$ & $0.06 \pm 0.1$ & NS \\
\hline & SRS & $36.3 \pm 16.4$ & $27.1 \pm 13.1$ & $9.3 \pm 3$ & $<0.01$ \\
\hline \multirow{3}{*}{ Frequency } & Whole bladder & $38.9 \pm 13.6$ & $40.4 \pm 13.7$ & $-1.4 \pm 2.4$ & NS \\
\hline & Prostatic urethra & $78.2 \pm 0.7$ & $78.2 \pm 0.6$ & $0.02 \pm 0.1$ & NS \\
\hline & SRS & - & - & - & - \\
\hline \multirow{3}{*}{ Incontinence } & Whole bladder & $38 \pm 12.6$ & $40.4 \pm 13.3$ & $-2.4 \pm 2.6$ & NS \\
\hline & Prostatic urethra & $78.2 \pm 1$ & $78 \pm 0.5$ & $0.2 \pm 0.5$ & NS \\
\hline & SRS & - & - & - & - \\
\hline \multicolumn{6}{|c|}{ Grade $\geq 2$} \\
\hline \multirow{3}{*}{ Retention } & Whole bladder & $42.2 \pm 13.3$ & $39.6 \pm 13.1$ & $2.6 \pm 3.5$ & NS \\
\hline & Prostatic urethra & $-0.3 \pm 0.3$ & $77.9 \pm 0.5$ & $78.1 \pm 0.6$ & NS \\
\hline & SRS & - & - & - & - \\
\hline \multirow{3}{*}{ Frequency } & Whole bladder & $38.6 \pm 12$ & $40.4 \pm 13.51$ & $-1.8 \pm 2.8$ & NS \\
\hline & Prostatic urethra & $78.5 \pm 0.6$ & $78 \pm 0.6$ & $0.5 \pm 0.2$ & NS \\
\hline & SRS & - & - & - & - \\
\hline \multirow{3}{*}{ Incontinence } & Whole bladder & $36.2 \pm 13.4$ & $40.3 \pm 13.1$ & $-4.1 \pm 4.9$ & NS \\
\hline & Prostatic urethra & $78.8 \pm 0.6$ & $77.9 \pm 0.6$ & $0.9 \pm 0.2$ & 0.07 \\
\hline & SRS & - & - & - & - \\
\hline
\end{tabular}

SRS: Symptom-related subregion; SD: Standard deviation, SE: Standard error; NS: not significant ( $p>0.05)$ 
Each SRS represents the subregion for the corresponding symptom (when identified). The Wilcoxon test has been used to calculate the $p$-values. The doses are not given for grade $\geq 2$ acute incontinence and hematuria and late dysuria and hematuria due to the absence or very low number of events. 
Table 2. Urinary toxicity prediction capability of the mean dose and the DVH for the whole bladder and the identified symptom-related subregions (SRSs) in the native space of the patients

\begin{tabular}{|c|c|c|c|c|c|c|c|c|}
\hline \multicolumn{9}{|c|}{ Acute toxicity (grade $\geq 1$ ) } \\
\hline \multirow[b]{2}{*}{ Endpoints } & \multirow[b]{2}{*}{ Regions } & \multicolumn{3}{|c|}{ Mean Dose } & \multicolumn{4}{|c|}{ DVH } \\
\hline & & $p$ value & OR $(95 \% \mathrm{Cl})$ & $\operatorname{AUC}(95 \% \mathrm{Cl})$ & $\begin{array}{l}\text { Most predictive DVH bin } \\
\text { (range of predictive bins)* }\end{array}$ & p value & OR $(95 \% \mathrm{Cl})$ & $\operatorname{AUC}(95 \% \mathrm{Cl})$ \\
\hline \multirow{2}{*}{ Retention } & $\begin{array}{l}\text { Whole } \\
\text { bladder }\end{array}$ & NS & - & - & V79 (V77-V79) & 0.052 & $1.06(1.01-1.13)$ & $0.60(0.51-0.67)$ \\
\hline & SRS & 0.04 & $1.06(1.01-1.2)$ & $0.62(0.56-0.68)$ & V72 (V63-V79) & 0.01 & $1.02(1.01-1.04)$ & $0.62(0.55-0.68)$ \\
\hline \multirow{2}{*}{ Incontinence } & $\begin{array}{l}\text { Whole } \\
\text { bladder }\end{array}$ & NS & - & - & - & NS & - & - \\
\hline & SRS & $<0.01$ & $2.1(1.27-3.43)$ & $0.74(0.66-0.81)$ & V80 (V80-V83) & 0.04 & $1.02(1.01-1.04)$ & $0.73(0.67-0.81)$ \\
\hline \multicolumn{9}{|c|}{ Late 5 -years toxicity (grade $\geq 1$ ) } \\
\hline \multirow[b]{2}{*}{ Endpoints } & \multirow[b]{2}{*}{ Regions } & \multicolumn{3}{|c|}{ Mean Dose } & \multicolumn{4}{|c|}{ DVH } \\
\hline & & $p$ value & HR $(95 \% \mathrm{Cl})$ & t-AUC (95\% Cl) & $\begin{array}{l}\text { Most predictive DVH bin } \\
\text { (range of predictive bins)* }\end{array}$ & p value & HR $(95 \% \mathrm{Cl})$ & t-AUC (95\% Cl) \\
\hline \multirow{2}{*}{ Dysuria } & $\begin{array}{l}\text { Whole } \\
\text { bladder }\end{array}$ & $<0.01$ & 1.05 (1.01-1.09) & $0.75(0.66-0.84)$ & V67 (V5- V78) & $<0.01$ & $1.03(1.01-1.05)$ & $0.72(0.63-0.82)$ \\
\hline & SRS & $<0.01$ & $1.1(1.04-1.17)$ & $0.8(0.71-0.88)$ & V52 (V32 - V76) & $<0.01$ & $1.05(1.02-1.08)$ & $0.81(0.72-0.90)$ \\
\hline \multirow{2}{*}{ Retention } & $\begin{array}{l}\text { Whole } \\
\text { bladder }\end{array}$ & $<0.01$ & $1.03(1.01-1.06)$ & $0.66(0.58-0.75)$ & V19 (V4-V78) & $<0.01$ & $1.02(1-1.04)$ & $0.67(0.59-0.75)$ \\
\hline & SRS & $<0.01$ & $1.04(1.02-1.05)$ & $0.71(0.63-0.78)$ & V35 (V7-V76) & $<0.01$ & $1.02(1.01-1.02)$ & $0.70(0.62-0.77)$ \\
\hline \multirow{2}{*}{ Hematuria } & $\begin{array}{l}\text { Whole } \\
\text { bladder }\end{array}$ & NS & - & - & V7 (V6-V18) & 0.04 & $1.04(1-1.07)$ & $0.65(0.55-0.75)$ \\
\hline & SRS & 0.04 & 1.02 (1.01-1.04) & $0.64(0.55-0.73)$ & V17 (V5-V25) & $<0.01$ & 1.02 (1.01-1.04) & $0.67(0.56-0.77)$ \\
\hline
\end{tabular}

SRS: Symptom-related subregion; OR: Odds ratio; HR: Hazard ratio; Cl: Confidence interval; AUC: Area under the ROC curve; t-AUC: time-dependent area under the ROC curve; NS: not significant ( $p>0.05) ;{ }^{*} \mathrm{DVH}$ bin with the highest AUC and range of bins with statistically significant $p$ value $(\leq 0.05)$ 
Univariate analysis was performed using logistic regression for acute toxicity and Cox regression for late toxicity. The predictive performance for acute toxicity was estimated using the area under the ROC curve. The predictive performance for late toxicity was estimated at 5 -years using the area under the timedependent ROC curve (t-AUC). Doses were not found predictive for grade $\geq 2$ toxicity. 